\title{
THE TENSION FIELD OF THE GAUSS MAP
}

\author{
BY \\ ERNST A. RUH( $\left.{ }^{1}\right)$ AND JAAK VILMS
}

\begin{abstract}
In this paper it is shown that the tension field of the Gauss map can be identified with the covariant derivative of the mean curvature vector field. Since a map with vanishing tension field is called harmonic the following theorem is ubtained as a corollary. The Gauss map of a minimal submanifold is harmonic.
\end{abstract}

1. Introduction. The study of 2-dimensional minimal surfaces in Euclidean $(2+p)$-space is greatly simplified by the fact that the Gauss map is antiholomorphic [1]. In this paper we will show that a weaker property holds for higher dimensions; namely that the Gauss map of a minimal submanifold of arbitrary codimension is harmonic.

A map of Riemannian manifolds is called harmonic if its tension field $\tau(f)$ vanishes [2]; equivalently, $f$ is an extremal of the energy integral $E(f)$ that generalizes the classical Dirichlet integral (see $\$ 3$ below). An immersion of a line as a geodesic, or more generally an immersion of a manifold as a minimal submanifold, is an example of a harmonic map.

In the case of a minimal submanifold in Euclidean space, we now have two harmonic maps, the immersion and the Gauss map. The harmonicity of the immersion has long been used to study minimal submanifolds. In [4] it has been shown that the harmonicity of the Gauss map has applications as well.

2. The Gauss map. In this section we review some of the properties of the Gauss map. Let $i: M \rightarrow R^{n+p}$ denote an immersion of an $n$-dimensional manifold into Euclidean $(n+p)$-space. The Gauss map associated to this immersion is the map $g: M \rightarrow G(n, p)$, where $G=G(n, p)$ denotes the Grassman manifold of $n$ planes in Euclidean $(n+p)$-space, and the image $g(x)$ of a point $x \in M$ is defined to be the tangent $n$-plane to $i(M)$ at $i(x)$. As usual, the Riemannian structure on $G(n, p)$ is defined by identifying $G(n, p)$ with the symmetric space $O(n+p) / O(n)$ $\times O(p)$, where $O(n+p)$ denotes the orthogonal group operating on the Euclidean space $R^{n+p}$.

The pull back under $g$ of the tangent bundle $T(G)$ to a bundle over $M$ is denoted by $g^{-1} T(G)$. In the following proposition, $T(M)$ and $N(M)$ will denote the tangent bundle and the normal bundle of $M$ in $R^{n+p}$ respectively.

Received by the editors June 23, 1969 and, in revised form, October 10, 1969.

AMS Subject Classifications. Primary 5345, 5374.

Key Words and Phrases. Codazzi equation, energy integral, harmonic map, immersed submanifold, mean curvature vector field, parallel mean curvature, tension field.

(1) Research partially supported by NSF GP 9521.

Copyright (C) 1970, American Mathematical Society 
Proposition. The bundle $g^{-1} T(G)$ is isometric to the tensor product $T(M)^{*}$ $\otimes N(M)$.

Proof. The isometry of the two fibres over a point $x \in M$ is defined to be the composition of the following three isometries.

$$
T_{g(x)}(G) \rightarrow \mathrm{g}(n, p) \rightarrow L\left(R^{n}, R^{p}\right) \rightarrow T_{x}(M)^{*} \otimes N_{x}(M) .
$$

The first arrow represents the standard identification of the tangent space of $G$ at $g(x)$ with the subspace $g(n, p)$ of the Lie algebra of $O(n+p)$. A basis of $g(n, p)$ is given by the set $\left(e_{i}^{*} \otimes e_{n+j}-e_{p+i}^{*} \otimes e_{j}, i=1, \ldots, n ; j=1, \ldots, p\right)$, where $e_{1}, \ldots$, $e_{n+p}$ is an orthogonal basis in $R^{n+p}$ and $e_{1}^{*}, \ldots, e_{n+p}^{*}$ is its dual basis. The second arrow represents the linear map that sends the basis element $e_{i}^{*} \otimes e_{n+j}-e_{p+i}^{*} \otimes e_{j}$ into $e_{i}^{*} \otimes e_{n+j}$, which is an element of the standard basis in the space of linear maps $L\left(R^{n}, R^{p}\right)$. The third arrow is obtained by choosing the basis $e_{1}, \ldots, e_{n+p}$ mentioned above to represent the point $g(x) \in G(n, p)$, i.e., by choosing $e_{1}, \ldots, e_{n}$ in $i_{*} T_{x}(M)$ and $e_{n+1}, \ldots, e_{n+p}$ in $N_{x}(M)$.

The main theorem will involve the mean curvature vector field $H$, a cross-section in the normal bundle $N(M)$. By definition $H$ is the trace of the second fundamental form (see [3]), which is a cross-section in $T(M)^{*} \otimes T(M)^{*} \otimes N(M)$. When we identify this bundle with $T(M)^{*} \otimes g^{-1} T(G)$ using the above proposition, the second fundamental form is identified with the tangent map $g_{*}$ of the Gauss map $g$. Thus $H=\operatorname{Tr} g_{*}$ is the trace of $g_{*}$ with respect to the Riemannian metric in $T(M)$. The immersion $i: M \rightarrow R^{n+p}$ induces a covariant derivative, $\nabla$, in $N(M)$. Using the above proposition again, $\nabla H$ can be identified with a cross-section in $g^{-1} T(G)$.

3. Harmonic maps. For the discussion of harmonic maps we will follow Eells and Sampson [2] closely. Let us consider a map $f: M_{1} \rightarrow M_{2}$ of Riemannian manifolds $\left(M_{1}\right.$ a manifold possibly with boundary $\left.\partial M_{1}\right)$, and define the following energy integral:

$$
E(f)=\frac{1}{2} \int_{M_{1}} \operatorname{Tr}\left\langle f_{*}, f_{*}\right\rangle d \sigma
$$

where $f_{*}$ denotes the differential of $f,\langle$,$\rangle denotes the metric on M_{2}$, and $\operatorname{Tr}$ denotes the trace of the quadratic form $X \mapsto\left\langle f_{*} X, f_{*} X\right\rangle$ with respect to the metric in $M_{1}$.

Now we want to recall a formula for the derivative of $E(f)$ with respect to a vector field along $f$. A vector field $v$ along $f$ is by definition a cross-section with compact support in the interior of $M_{1}$, of the bundle $f^{-1} T\left(M_{2}\right)$. To compute the derivative we embed $f$ in a one-parameter family of maps $f_{t}: M_{1} \rightarrow M_{2}$, where $f_{t}(x)$ is the endpoint of a geodesic segment starting at $f(x)$ determined in length - and direction by $v(x)$. If the derivative of $E\left(f_{t}\right)$ with respect to $t$ at $t=0$ is denoted by $E^{\prime}(f)$, then the following formula holds.

$$
E^{\prime}(f)=-\int_{M_{1}}\langle\tau(f), v\rangle d \sigma
$$


where $\tau(f)$, a cross-section in $f^{-1} T\left(M_{2}\right)$, is called the tension field of $f$. In [2] it has been shown that the above formula, required to hold for all smooth cross-sections in $f^{-1} T\left(M_{2}\right)$, characterizes $\tau(f)$ uniquely. The equation $\tau(f)=0$, a generalized Laplace equation, is the Euler-Lagrange equation of the energy integral. Since $\tau(f)=0$ defines a harmonic map, we see $f$ is harmonic iff it is an extremal of $E(f)$ with respect to the variations $v$.

4. The main results. In this section, $g: M \rightarrow G(n, p)=G$ will denote the Gauss map associated to an immersion $i: M \rightarrow R^{n+p}$. The tension field of $g, \tau(g)$, a crosssection of $g^{-1} T(G)$, has been defined in $\S 3$. The following theorem will also involve the covariant derivative, $\nabla H$, of the mean curvature vector field $H$. As explained in $\S 2, \nabla H$ may be identified with a cross-section of $g^{-1} T(G)$.

THEOREM. $\tau(g)=\nabla H$.

COROLlARY I. If $M$ is immersed with parallel mean curvature vector field $H$, then the Gauss map $g: M \rightarrow G$ is harmonic.

If the codimension of the immersion is one, then parallel mean curvature simply means constant mean curvature. For this special case the above results have been obtained in [4].

If the manifold $M$ is immersed as a minimal submanifold, then the mean curvature vector field $H$ and its covariant derivative $\nabla H$ are zero. This observation yields the following result mentioned in the introduction.

COROLlARY II. The Gauss map of a minimal $n$-dimensional submanifold in $R^{n+p}$ is harmonic.

5. Proof of the theorem. In this section we compute the tension field $\tau(g)$ of the Gauss map $g$. This will be done by computing the first variation $E^{\prime}(g)$ of the energy integral $E(g)$ in the direction of a vector field $v$ along $g$ and then comparing the result with the defining equation for $\tau(g)$.

For this purpose we will define a one-parameter family of maps $f_{t}: M \rightarrow G(n, p)$ associated to a vector field $v$ along $g$. The representative of the coset $f_{t}(x)$ is defined to be the product $(\exp t v) g(x)$ in $O(n+p)$. The exponential exp $t v$ makes sense if, as explained in $\S 2$, the vector $v(x)$ is identified with an element in $g(n, p)$. For convenience $g(x)$ is represented by the following element in the orthogonal group. Let $e_{1}, \ldots, e_{n+p}$ be the moving frame obtained by parallel translation along geodesics in $M$ of a frame at $x_{0} \in M$. Now $g(x)$ is represented by the linear map that sends the frame at $x_{0}$ into the frame at a nearby point $x \in M$.

For the moment we think of $f_{t}$ as a map $M \rightarrow \mathrm{GL}(n+p, R)$. This will be useful for the computation of $E^{\prime}(g)$. The differential $f_{t *}$ is equal to $(I+t v+\cdots)_{*} g$ $+(I+t v+\cdots) g_{*}$. Evaluating $f_{t *}$ at $x_{0}$, where $g$ is equal to the identity $I$, and neglecting higher order terms in $t$ we obtain $f_{t *}=t D v+g_{*}$, where $D v$ is the derivative of 
$v: M \rightarrow \mathrm{GL}(n+p, R)$. The linear term in $t$ of $\operatorname{Tr}\left\langle f_{*}, f_{*}\right\rangle$ is equal to $2 t \operatorname{Tr}\left\langle g_{*}, D v\right\rangle$. Since the image under $g_{*}$ lies in $g(n, p)$ the term $D v$ may be replaced by its projection to $\mathrm{g}(n, p)$.

So far, the range of $g_{*}$, the projection of $D v$, and the metric $\langle,>$ have been objects related to the bundle $g^{-1} T(G)$. Now we use the proposition of $\S 2$ to replace these objects by their counterparts related to $T(M)^{*} \otimes N(M)$. Thus the projection of $D v$ to $\mathrm{g}(n, p)$ is replaced by $\nabla v$, the covariant derivative of the cross-section $v$ of $T(M)^{*} \otimes N(M)$. This is verified by observing that, due to our choice of the moving frame $e_{1}, \ldots, e_{n+p}$ made earlier, both the projection of $D\left(e_{i}^{*} \otimes e_{n+j}-e_{p+i}^{*} \otimes e_{j}\right)$ to $g(n, p)$ and the covariant derivative $\nabla\left(e_{i}^{*} \otimes e_{n+j}\right)$ in $T(M)^{*} \otimes N(M)$ are zero at $x=x_{0}$.

The derivative of the energy integral for $t=0$ can therefore be computed in terms of the bundle $T(M)^{*} \otimes N(M)$. In fact we obtain

$$
E^{\prime}(g)=\int_{M} \operatorname{Tr}\left\langle g_{*}, \nabla v\right\rangle d \sigma .
$$

In the next paragraph we perform integration by parts to give $E^{\prime}(g)$ a more useful expression.

First, we observe that

$$
\operatorname{Tr}\left\langle g_{*}, \nabla v\right\rangle=\operatorname{Tr} \nabla\left\langle g_{*}, v\right\rangle-\operatorname{Tr}\left\langle\nabla g_{*}, v\right\rangle
$$

because $\nabla<,>=0$. Second, we observe that

$$
\operatorname{Tr}\left\langle g_{*}, v\right\rangle d \sigma=d *\left\langle g_{*}, v\right\rangle
$$

where $*$ is the star operator on exterior forms and $d$ is the exterior derivative. Third, $\operatorname{Tr}\left\langle\nabla g_{*}, v\right\rangle$ is shown to be equal to $\langle\nabla H, v\rangle$ as follows. Under the identification of the proposition of $\S 2, g_{*}$ is a cross-section in $T(M)^{*} \otimes T(M)^{*} \otimes N(M)$. $\nabla g_{*}$ therefore is a cross-section in $T(M)^{*} \otimes T(M)^{*} \otimes T(M)^{*} \otimes N(M)$. The symmetry of $g_{*}$ with respect to the factors in $T(M)^{*}$, together with the Codazzi equation [3, p. 25, Proposition 4.3] implies that $\nabla g_{*}$ is symmetric in all three factors $T(M)^{*}$. We may therefore take the trace $\operatorname{Tr} \nabla g_{*}$ with respect to any two factors in $T(M)^{*}$. Furthermore, since the operations $\operatorname{Tr}$ and $\nabla$ commute, we obtain $\operatorname{Tr} \nabla g_{*}=\nabla H$, where $H=\operatorname{Tr} g_{*}$, as explained in $\S 2$.

The above three observations imply that

$$
E^{\prime}(g)=\int_{M} d *\left\langle g_{*}, v\right\rangle-\int_{M}\langle\nabla H, v\rangle d \sigma
$$

The first integral, by Stokes' theorem, is zero because $v$ is zero on the boundary $\partial M$. Comparing the formula for $E^{\prime}(g)$ with the defining equation for $\tau(g)$ of $\S 3$ we obtain $\tau(g)=\nabla H$, which was to be shown. 


\section{BIBLIOGRAPHY}

1. S. S. Chern, Minimal surfaces in a Euclidean space of $N$ dimensions, Differential and Combinatorial Topology (A Symposium in Honor of Marston Morse), Princeton Univ. Press, Princeton, N. J., 1965, pp. 187-198. MR 31 \#5156.

2. J. Eells, Jr. and J. H. Sampson, Harmonic mappings of Riemannian manifolds, Amer. J. Math. 86 (1964), 109-160. MR 29 \#1603.

3. S. Kobayashi and K. Nomizu, Foundations of differential geometry. Vol. II, Interscience Tracts in Pure and Appl. Math., no. 15, Interscience, New York, 1969.

4. E. A. Ruh, Asymptotic behaviour of non-parametric minimal hypersurfaces, J. Differential Geometry (to appear).

Purdue University,

LAFAYETTE, INDIANA 47907 